\title{
Presencia, abundancia y asociatividad de Citronella mucronata en bosques secundarios de Nothofagus obliqua en la precordillera de Curicó, región del Maule, Chile
}

\author{
Presence, abundance and associability of Citronella mucronata in secondary forests dominated by \\ Nothofagus obliqua in the foothills of Curicó, Maule Region, Chile
}

\author{
Patricio Corvalán $^{\text {a*, }}$ Mauricio Galleguillos ${ }^{\text {, }}$, Jaime Hernández ${ }^{\text {a }}$ \\ *Autor de correspondencia: a Universidad de Chile, Facultad de Ciencias Forestales y de la Conservación de la Naturaleza, \\ Departamento de Gestión Forestal y de su Medioambiente, Casilla 9206, Santiago, Chile, tel.: 56-2-5418834, pcorvala@uchile.cl \\ b Universidad de Chile, Facultad de Ciencias Agronómicas, Departamento de Ciencias Ambientales y Recursos Naturales \\ Renovables, Santiago, Chile.
}

\begin{abstract}
SUMMARY
In Chile, Citronella mucronata is a tree species classified as "nearly endangered", highlighting the lack of quantitative information about their populations. The aim of this study was to provide knowledge about $C$. mucronata populations in secondary forests of Nothofagus obliqua in the Andes foothills of Maule region, central Chile. Specifically the goal was to examine the presence, abundance and associability of C. mucronata and its relation to landscape variables of the Andes foothills. A forest inventory was conducted with the setup of 197 clusters with five plots each. Moreover, a computation of a high resolution geomorphological index was obtained based on LiDAR remote sensing, which allowed establishing geographical distribution patterns of the species. In the sampling area, C. mucronata was present in $31.1 \%$, the average abundance was 117 trees per hectare and the mean altitudinal distribution was $1.149 \mathrm{~m}$ a.s.l. with a $142 \mathrm{~m}$ interquartile range of the altitude reached preferably in well-drained areas as evidenced by the landform index values. Citronella mucronata was slightly associated with Aextoxicon punctatum, Aristotelia chilensis, Austrocedrus chilensis, Azara petiolaris, Cryptocarya alba, Laurelia sempervirens, Lomatia dentata, Luma apiculata, Maytenus boaria, Nothofagus obliqua, Persea lingue, Podocarpus salignus and Quillaja saponaria and not associated with Drimys winteri, Escallonia pulverulenta, Lithraea caustica, Lomatia hirsuta or Myrceugenia exsucca. Their density shows no significant correlation with the density of any other species. The study allowed deepening knowledge of the species in secondary forests of $N$. obliqua.
\end{abstract}

Key words: native forest, landform index, terrain digital elevation models, nearly endangered species.

\section{RESUMEN}

En Chile, Citronella mucronata es un árbol clasificado como "casi amenazado", destacándose la falta de información cuantitativa de sus poblaciones. El objetivo de este estudio fue aportar conocimiento sobre las poblaciones de C. mucronata en bosques secundarios de Nothofagus obliqua en la precordillera del Maule, Chile central. Específicamente, se examinó la presencia, abundancia y asociatividad de la especie y su relación con variables del paisaje de la precordillera andina. Se realizó un inventario forestal a partir de la instalación de 197 conglomerados de cinco parcelas cada uno. Además se realizó un cálculo de un índice geomorfológico de alta resolución obtenido por teledetección LiDAR, lo que permitió establecer la distribución geográfica de la especie. En el área de estudio, la presencia de árboles adultos de C. mucronata fue 31,1 \%, la densidad fue 117 árboles ha-1 ${ }^{-1}$ la altitud media fue de 1149 m s.n.m. con un rango intercuartilíneo de la distribución altitudinal de 142 m y se ubicó de preferencia en terrenos bien drenados. Citronella mucronata se asoció levemente a Aextoxicon punctatum, Aristotelia chilensis, Austrocedrus chilensis, Azara petiolaris, Cryptocarya alba, Laurelia sempervirens, Lomatia dentata, Luma apiculata, Maytenus boaria, Nothofagus obliqua, Persea lingue, Podocarpus salignus y Quillaja saponaria. No se asoció a Drimys winteri, Escallonia pulverulenta, Lithraea caustica, Lomatia hirsuta ni a Myrceugenia exsucca. La densidad de C. mucronata no presentó correlación significativa con la densidad de ninguna otra especie. El estudio permitió profundizar el conocimiento sobre la distribución de la especie en bosques secundarios de N. obliqua.

Palabras clave: bosque nativo, índice de geoforma, modelo de elevación digital de terreno, especie casi amenazada.

\section{INTRODUCCIÓN}

En Chile, la prohibición de corta y alteración del hábitat de especies forestales autóctonas incluidas en las categorías "En Peligro Crítico, En Peligro, Vulnerable, Casi Amenazada y Preocupación Menor” está regulada por la ley 20.417 promulgada en enero de 2010 y actualmente vigente. Esta fue modificada para satisfacer los criterios de la International Union for Conservation of Nature (IUCN) (MMA 2013). En particular, Citronella mucronata (Ruiz et Pav.) D. Don, naranjillo, está clasificada como especie "Casi amenazada", categoría definida en el decreto Supre- 
mo 13 de 2013 del Ministerio del Medioambiente de Chile (MMA) y que se refiere a cuando "habiendo sido evaluada, no satisface actualmente, los criterios para las categorías En Peligro Crítico, En Peligro o Vulnerable; pero está próxima a satisfacer los criterios de estos últimos, o posiblemente los satisfaga, en el futuro cercano" (MMA 2013). De acuerdo al MMA (2013), C. mucronata se distribuye en forma amplia pero discontinua en las cordilleras de los Andes y la Costa desde la región de Coquimbo (provincia de Limarí, 3040’ S) hasta la región de Los Lagos (provincia de Osorno $40^{\circ} 30^{\prime} \mathrm{S}$ ). Se encuentra tanto en la Cordillera de los Andes como de la Costa. También se reconoce no disponer de antecedentes cuantitativos de la cantidad ni el tamaño de sus poblaciones; sin embargo, se señala que "existe información cualitativa que indica que raramente se encuentran rodales a lo largo de su distribución", aunque se mencionan 54 poblaciones. Finalmente el MMA (2013) señala que la especie es probablemente frecuente, pero con bajas abundancias poblacionales locales.

Donoso (1981a) indica que C. mucronata crece en el bosque esclerófilo, con individuos aislados o en pequeños bosquetes sobre laderas sombrías y sectores húmedos. Más tarde, Donoso (1990) describe a esta especie como tolerante a la sombra que regenera exclusivamente en el interior del bosque. Gajardo (1994) incluye a C. mucronata en las regiones del matorral y bosque esclerófilo y del bosque caducifolio. En un estudio posterior, Hechenleiter et al. (2005) localizan cartográficamente las 54 poblaciones dentro de su distribución y para la región andina del Maule las mencionan en las localidades de Radal Siete Tazas y Altos de Lircay. Según el MMA (2012), existe escasa información sobre el hábitat de la especie, siendo reportada en sectores húmedos o fondos de quebradas en zonas de baja altitud de la zona centro-sur de Chile. En el tipo forestal roble-hualo, correspondiente a Nothofagus obliqua y Nothofagus glauca respectivamente, la presencia de $C$. mucronata es frecuente y dependiendo de la latitud, puede asociarse a Nothofagus dombeyi (Mirb.) Bl. y Nothofagus alpina (Poepp. et Endl.) Oerst. (MMA 2012). Este tipo forestal ha sido ampliamente alterado durante los últimos siglos generando bosques secundarios o renovales debido a tala para obtención de leña y madera y habilitación de terrenos para la agricultura los que han generado procesos de sucesión secundaria (Donoso 1993). En estos bosques se presenta según Donoso (1993) "un verdadero mosaico de rodales de distintas edades derivados de la acción antrópica". Finalmente, su presencia ha sido señalada en estudios de diversidad de zonas cercanas dentro del sur de Chile (Altamirano et al. 2010, 2013) y en bosques secos de la zona central con datos cuantitativos de su distribución y abundancia (Hechenleiter et al. 2005, Newton et al. 2011).

De particular interés resulta incorporar las geoformas correspondientes a fondos de valle, al ser relevantes en la determinación de características funcionales de la hidrología y la geomorfología de los ecosistemas y de las cuencas. De esta manera, y de la separación entre geoformas cóncavas (fondo de valle) y aquellas convexas (colinas o pendientes) emergen diferencias fundamentales en los factores de formación de suelo y en la capacidad de retención hídrica. Las características de los suelos constituyen un elemento clave para determinar el paisaje. Por lo señalado se plantea que las geoformas del terreno pueden ser de gran relevancia para explicar los patrones de distribución de las especies (Hoersh et al. 2002), en particular de aquellas como C. mucronata que se caracterizarían por su preferencia de habitar sectores húmedos (Donoso 1981a, Hechenleiter et al. 2005).

El objetivo de este estudio se enmarca en aportar conocimiento sobre las poblaciones de $C$. mucronata en bosques secundarios de $N$. obliqua en la precordillera del Maule, Chile central. Específicamente, se estableció examinar empíricamente la presencia, abundancia y asociatividad de C. mucronata y su relación con variables del paisaje obtenidas mediante teledetección LiDAR.

\section{MÉTODOS}

Área de estudio. El área de estudio se localiza en los contrafuertes cordilleranos donde termina el valle del estero Upeo ( $35^{\circ} 07^{\prime} \mathrm{S}, 70^{\circ} 56^{\prime} \mathrm{O}$; 698 m s.n.m.), cubre 1.295 hectáreas, en exposición sur y topografía abrupta con mesetas interiores y esta conformada por dos cuencas hidrográficas que drenan en el estero Upeo por el límite sur (figura 1). El clima presenta en promedio una temperatura anual de $12,3{ }^{\circ} \mathrm{C}$, una precipitación anual de $1.315 \mathrm{~mm}$, con un periodo de 216 días libres de heladas, y una humedad relativa media de 68 \% (Santibáñez y Uribe 1993). Los suelos corresponden a la Asociación Sierra Bellavista, Mollic Haploxeralf (Alfisol), desarrollado de materiales volcánicos (andesita) y en algunos sectores recubierto por cenizas volcánicas frescas, en pendientes de 30 a $50 \%$ (CIREN 1996). En los sectores planos la profundidad del suelo es superior a $1 \mathrm{~m}$ disminuyendo fuertemente a mayor altitud (sobre los $1.400 \mathrm{~m}$ s.n.m.). La presencia de trumao profundo permite el almacenamiento y drenaje del agua en los esteros, aún en los meses más críticos de verano y comienzo de otoño. La vegetación natural corresponde a la región del bosque caducifolio de La Montaña, que por el contrafuerte cordillerano andino se distribuye desde el sur de la región del Libertador Bernardo O'Higgins hasta el norte del río Biobío (Gajardo 1994). Esta formación vegetal se caracteriza por su gran riqueza florística y el límite norte de algunas especies leñosas y herbáceas más características de los bosques australes. Las comunidades características de esta formación son Nothofagus obliqua (Mirb.) Oerst. - Ribes punctatum Ruiz et Pav. y Austrocedrus chilensis (D. Don) Pic. Serm. et Bizzarri (Gajardo 1994). La zona se inserta en el piso de vegetación denominado "Bosque caducifolio mediterráneo andino de Nothofagus obliqua y Austrocedrus chilensis". Este piso de vegetación se distribuye en laderas medias de la Cordillera de Los Andes de la región del Maule y norte del Biobío, entre los 


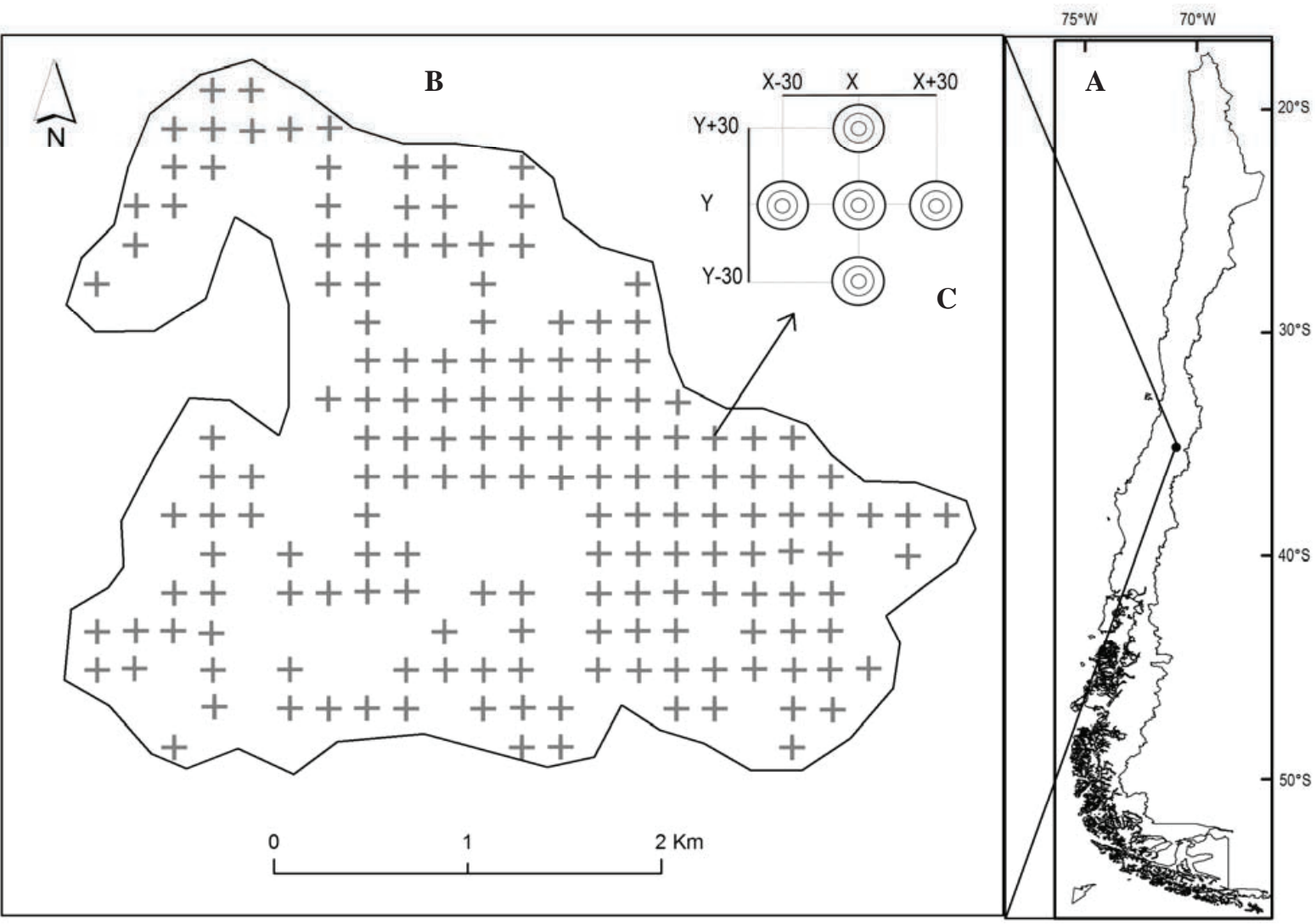

Figura 1. A) Ubicación del sitio de estudio, B) configuración de las unidades de muestreo del inventario forestal y C) configuración de las unidades de registro. Los círculos concéntricos indican radios de 2, 4 y 8 m y las coordenadas X e Y los centros de las parcelas en coordenadas UTM (m).

A) Localization of the study site, B) configuration of the sampling units of the forest inventory and C) configuration of the registry units. The concentric circles indicate radii of 2, 4 and 8 meters and X and Y coordinates indicate the centers of the plots in UTM (m).

900 y 1.800 m s.n.m. Corresponde a un bosque caducifolio con participación de ciprés de la cordillera Austrocedrus chilensis y especies esclerófilas, principalmente Lithrea caustica (Molina) Hook. et Arn. y Quillaja saponaria Mol (Luebert y Pliscoff 2006).

La elección del área de estudio se determinó considerando las grandes variaciones de altitud, exposición y pendiente además de incluir tres tipos forestales (esclerófilo, roble-hualo y ciprés de la cordillera) lo cual permite capturar, describir y cuantificar posibles escenarios de manejo donde C. mucronata se presenta. Se restringió el área a las condiciones de intervención que la ley permite (menores a $60 \%$ de pendiente y estar fuera de los cursos de agua).

Para caracterizar el hábitat de C. mucronata y sus especies asociadas, se utilizan las variables altitud y un índice de geoforma.

Diseño muestral. Para medir la presencia, abundancia y asociatividad de $C$. mucronata se realizó un inventario forestal mediante conglomerados de parcelas o unidades de muestreo distribuidos sistemáticamente en una grilla sobre el área, con partida al azar. El conglomerado estu- vo constituido por hasta cinco unidades de muestreo cada uno, que correspondieron a parcelas circulares concéntricas de 2, 4 y $8 \mathrm{~m}$ de radio, con medición de árboles según el diámetro a la altura de pecho (DAP) mayor o igual a 5, 10 y $20 \mathrm{~cm}$, respectivamente (figura 1). Para cuantificar la presencia, abundancia y asociatividad de C. mucronata, sólo se consideró el estrato arbóreo teniendo en cuenta la determinación de una cota mínima para estas variables El conglomerado estuvo constituido por una parcela central y cuatro parcelas satélites ubicadas en dirección N, S, E, y O. Entre los centros de las parcelas satélites y la parcela central la distancia fue de $30 \mathrm{~m}$, y entre los centros de conglomerados la separación fue de $200 \mathrm{~m}$. en las mismas direcciones señaladas El tamaño total de la muestra fue de 814 parcelas, agrupadas en 176 conglomerados, cada uno de los cuales representa aproximadamente una hectárea. Cuando alguna de las unidades de registro se presentó en una pendiente superior a $60 \%$ o en un curso de agua fue eliminada del conglomerado e igualmente si dos o más cumplían esa condición, Tanto la ubicación de las parcelas como la posición altitudinal se midieron con GPS (Garmin Legend $\mathrm{HCx}$ ). En este trabajo se consideró presencia 
como la cantidad relativa de conglomerados donde la especie se presentó, siendo efectiva cuando existía al menos un individuo en un conglomerado y abundancia como la frecuencia o densidad de árboles por hectárea.

Para caracterizar la forma o concentración de frecuencias de la densidad de árboles de C. mucronata se utilizó el sesgo o asimetría de Fisher (D’Ottone 1971). Se midió el nivel de asociatividad entre la densidad de árboles de $C$. mucronata y el resto de las especies a través del coeficiente de correlación de Spearman en los conglomerados en los cuales se encontraba presente. Las correlaciones nulas (cero) se producen cuando alguna de las especies en evaluación no tiene presencia. Las correlaciones fueron positivas cuando la densidad de individuos por especie se ordenaba en forma similar y negativas cuando el orden era inverso (D’Ottone 1971). La significación de la correlación se estableció cuando el coeficiente de correlación de Spearman era diferente de cero con un nivel de confiabilidad del $95 \%$.

Para obtener las variables de geoforma del paisaje se utilizó información de un vuelo LiDAR (Harrier 54/G4 Dual System) permitiendo obtener una nube de puntos de pulso discreto con un promedio de 4,64 puntos $\mathrm{m}^{2}$, la cual permitió obtener un modelo de elevación digital (DEM Digital Elevation Model), con características de un modelo de elevación de terreno. Para el procesamiento de la nube de puntos LiDAR, se utilizó el software TreesVIS (Weinacker et al. 2004) generando un DEM de 10 metros de resolución. Este DEM fue analizado utilizando CLASS Spatial Analyst 2.0.1 (Teng et al. 2005, 2008) para extraer atributos digitales de terreno primarios, secundarios y terciarios. CLASS fue diseñado como una herramienta hidrológica para operar a escala de cuencas hidrográficas. Este programa permite el cálculo de Multi-resolution Valley Bottom Flatness (MrVBF) (Gallant y Dowling 2003), y se basa en la identificación de fondos de valle los cuales están definidos por los siguientes supuestos: (1) los fondos de valle son bajos y planos en comparación a lo que lo rodea; (2) los fondos de valle existen en un rango de escalas; (3) los fondos de valle anchos son más planos que aquellos más estrechos. En síntesis, se puede señalar que para realizar el cálculo de MrVBF, se considera entonces cuan bajo y plano es el fondo de valle. Para estimar el grado de planicidad se utiliza el inverso de la pendiente y para estimar cuan bajo es el valle se utiliza una clasificación de las elevaciones con respecto a un ventana circular de $3 \mathrm{x}$ 3 celdas. Ambas mediciones son normalizadas entre 0 y 1 , combinadas y posteriormente interpretadas como una matriz de confusión. De esta manera la identificación de fondos de valle es llevada a cabo considerando múltiples resoluciones calculadas mediante un re-muestreo del DEM hacia resoluciones más gruesas y repitiendo el proceso iterativamente. Así el índice MrVBF es entonces una combinación ponderada de valores individuales de valles planos. Para el cálculo del índice se requiere un importante número de pasos los cuales están descritos en detalle en Gallant y Dowling (2003). Estos vienen incluidos en el software CLASS Spatial Analyst 2.0.1 (Teng et al. 2005).
Para el procesamiento y cálculo de estadísticos, los datos fueron procesados utilizando los programas XLSTAT2012, ArcGIS 9.3 y R-project, los que permitieron obtener estadísticas de tendencia central y dispersión para realizar las comparaciones con otras especies.

\section{RESULTADOS}

Presencia y abundancia. Citronella mucronata se presentó como la sexta especie de mayor presencia con un 31,1\% de la muestra, siendo superada por otras cinco entre ellas Podocarpus saligna (D. Don) (33,5 \%), Azara petiolaris (D. Don.) Johnst. (56,3 \%), Lomatia dentata (Ruiz et Pavón) R. Br. (65,3 \%) y Nothofagus obliqua (99,4 \%) (cuadro 1). Contrariamente, las especies de menor presencia fueron Escallonia pulverulenta (Ruiz et Pav.) Pers. y Lithraea caustica (1,8 \%), Myrceugenia exsucca (DC) Berg. (2,4 \%), Drimys winteri J. R. et G. Forster (5,4 \%), Persea lingue Nees. y Lomatia hirsuta (Lam.) Diels. ex Macbr. (6,0 \%) y Maytenus boaria Mol. (9,6 \%). En el área de estudio, C. mucronata presentó una baja densidad $\left(36,4\right.$ árboles $\left.\mathrm{ha}^{-1}\right)$, pero este valor fue muy superior al de otras especies como Myrceugenia exsucca, Drimys winteri, Escallonia pulverulenta y Lithraea caustica que presentaron densidades menores a 10 árboles ha ${ }^{-1}$. A su vez, C. mucronata presentó una densidad inferior a Aristotelia chilensis (Mol.) Stuntz, Aextoxicon punctatum Ruiz et Pavón. y Cryptocarya alba (Mol.) Looser que tuvieron densidades entre 100 a 200 árboles ha ${ }^{-1}$, y a la de las especies más abundantes como Azara petiolaris, Lomatia dentada y Nothofagus obliqua, que tuvieron densidades entre 250 a 1.000 árboles ha $^{-1}$ (cuadro 1). Considerando sólo la abundancia de cada una de las especies presentes del área, $C$. mucronata tuvo una densidad media de 117 árboles ha-1, ubicándose entre las menos abundantes junto a Myrceugenia exsucca (23 árboles ha-1 ${ }^{-1}$ y Drimys winteri (56 árboles ha $\left.^{-1}\right)$ y lejos de aquellas más abundantes como Aristotelia chilensis (583, árboles ha ${ }^{-1}$ ), Aextoxicon punctatum (662 árboles ha-1) y Nothofagus obliqua (955 árboles ha-1). Cabe destacar que la abundancia cambió en función de si se consideró la superficie total muestreada o el área de distribución donde cada especie se encontró presente (cuadro 1). A modo de ejemplo, C. mucronata tuvo una densidad media de 36 árboles ha- ${ }^{-1}$ si se considera el área total y de 117 árboles ha ${ }^{-1}$ cuando se consideró el área de distribución donde se encontró presente.

Distribución altitudinal. Citronella mucronata presentó una amplia distribución altitudinal desde los 682 y hasta los 1.366 m s.n.m., cubriendo un 96 \% del rango (cuadro 1, figura 2) y se situó como una de las especies cuyo valor medio ocupó las mayores posiciones altitudinales junto a Myrceugenia exsucca, Drimys winteri, Azara petiolaris y Aristotelia chilensis, y contrariamente a otras de menor preferencia de elevación (bajo los 1.000 m s.n.m.) como Lithraea caustica, Persea lingue, Lomatia hirsuta, Austro- 
Cuadro 1. Presencia porcentual en relación al total de conglomerados y valores promedio de abundancia, altitud e índice MrVBF* de las especies arbóreas con DAP $>5 \mathrm{~cm}$. Entre paréntesis se describe la proporción del recorrido intercuartilíneo sobre el rango de amplitud de su distribución en porcentaje.

Percentage relative to the total presence of clusters and mean abundance, altitude and $\mathrm{MrVBF} *$ tree species with $\mathrm{DBH}>5 \mathrm{~cm}$ index values. In brackets, the proportion of travel on the interquartile range of amplitude distribution in percentage is described.

\begin{tabular}{|c|c|c|c|c|c|}
\hline \multirow{2}{*}{ Especie } & \multirow{2}{*}{ Presencia (\%) } & \multicolumn{2}{|c|}{ Abundancia (árboles ha"-1) } & \multirow{2}{*}{$\begin{array}{l}\text { Altitud } \\
\text { (m s.n.m.) }\end{array}$} & \multirow{2}{*}{$\mathrm{MrVBF}$} \\
\hline & & $\begin{array}{l}\text { En área de } \\
\text { estudio }\end{array}$ & $\begin{array}{l}\text { En área de } \\
\text { distribución }\end{array}$ & & \\
\hline Aextoxicon punctatum & 19,8 & $131(0,0)$ & $662(20,7)$ & $1.031(45,1)$ & $0,325(25,5)$ \\
\hline Aristotelia chilensis & 21,0 & $122(0,0)$ & $583(26,7)$ & $1.186(16,8)$ & $0,223(8,8)$ \\
\hline Austrocedrus chilensis & 11,4 & $42(0,0)$ & $369(13,8)$ & $889(36,6)$ & $0,151(32,7)$ \\
\hline Azara petiolaris & 56,3 & $259(12,1)$ & $461(19,1)$ & $1.168(25,3)$ & $0,265(17,6)$ \\
\hline Citronella mucronata & 31,1 & $36(2,0)$ & $117(13,2)$ & $1.149(20,8)$ & $0,111(7,1)$ \\
\hline Cryptocarya alba & 29,3 & $142(4,1)$ & $484(30,9)$ & $992(50,6)$ & $0,110(9,2)$ \\
\hline Drimys winteri & 5,4 & $3(0,0)$ & $56(46,5)$ & $1.158(36,9)$ & $0,934(80,0)$ \\
\hline Escallonia pulverulenta & 1,8 & $3(0,0)$ & $172(50,0)$ & $959(50,0)$ & $0,013(50,0)$ \\
\hline Laurelia sempervirens & 12,6 & $21(0,0)$ & $166(28,1)$ & $1.128(17,1)$ & $0,405(28,2)$ \\
\hline Lithraea caustica & 1,8 & $6(0,0)$ & $345(50,0)$ & $767(50,0)$ & $0,045(50,0)$ \\
\hline Lomatia dentata & 65,3 & $305(22,3)$ & $467(26,8)$ & $1.133(21,9)$ & $0,260(17,7)$ \\
\hline Lomatia hirsuta & 6,0 & $24(0,0)$ & $404(35,4)$ & $852(11,1)$ & $0,154(14,5)$ \\
\hline Luma apiculata & 14,4 & $80(0,0)$ & $559(21,9)$ & $1.087(35,8)$ & $0,507(35,7)$ \\
\hline Maytenus boaria & 9,6 & $12(0,0)$ & $124(38,2)$ & $1.049(26,0)$ & $0,381(37,1)$ \\
\hline Myrceugenia exsucca & 2,4 & $1(0,0)$ & $23(25,0)$ & $1.155(35,5)$ & $0,926(43,6)$ \\
\hline Nothofagus obliqua & 99,4 & $949(19,4)$ & $955(18,7)$ & $1.126(26,1)$ & $0,252(17,8)$ \\
\hline Persea lingue & 6,0 & $19(0,0)$ & $316(67,9)$ & $822(14,4)$ & $0,256(56,1)$ \\
\hline Podocarpus saligna & 33,5 & $51(1,8)$ & $153(13,5)$ & $1.136(18,5)$ & $0,370(24,1)$ \\
\hline Quillaja saponaria & 26,9 & $39(1,3)$ & $144(22,6)$ & $1.078(44,9)$ & $0,204(14,5)$ \\
\hline Total & 100,0 & $2.249(33,3)$ & $2.249(33,3)$ & $1.124(26,4)$ & $0,252(17,8)$ \\
\hline
\end{tabular}

* Multi-resolution Valley Bottom Flatness.

cedrus chilensis y Escallonia pulverulenta. A su vez, C. mucronata presentó uno de los mayores rangos altitudinales junto con Nothofagus obliqua y Lomatia dentata en contraposición a Myrceugenia exsucca, Escallonia pulverulenta y Lithraea caustica. Considerando el recorrido intercuartilíneo (D’Ottone 1971), C. mucronata concentró la mitad de su población entre las altitudes de 1.098 y 1.240 m s.n.m., representando sólo el 20,8 \% de la amplitud de su distribución local. Para esta área, Lomatia hirsuta, Persea lingue, Aristotelia chilensis, Laurelia sempervirens (Ruiz et Pavón) Tul. y Podocarpus salignus, fueron especies aún más concentradas altitudinalmente y, por el contrario, QuiIlaja saponaria, Aextoxicon punctatum, Lithraea caustica y Cryptocarya alba, presentaron una mayor dispersión entorno a su valor central (figura 2). Si se considera el sesgo o asimetría de Fisher de la distribución, C. mucronata tuvo un valor de -1,2 indicando una mayor concentración de la población por sobre la cota media al igual que la mayo- ría de las especies, a excepción de Austrocedrus chilensis, Nothofagus obliqua, Persea lingue y Lomatia hirsuta.

Índice de geoforma. Considerando el MrVBF como un indicador indirecto de drenaje, C. mucronata se encontró entre las especies con los menores valores $(0,111)$, un poco superior al de Escallonia pulverulenta $(0,013)$, Lithraea caustica $(0,045)$ y levemente superior a Cryptocarya alba $(0,110) \mathrm{y}$, muy distante e inferior que Luma apiculata (DC.) Burret, Myrceugenia exsucca y Drimys winteri cuyos índices promedio fueron $0,507,0,926$ y 0,934, respectivamente (cuadro 1, figura 3). Con respecto a la interpretación del índice MrVBF, se observaron algunos patrones a considerar para C. mucronata: (1) valores del índice MrVBF cercanos a 0 siendo una de las especies que presentó los valores más bajos junto a Cryptocarya alba, Lithraea caustica y Escallonia pulverulenta, y (2) rango intercuartilíneo estrecho, indicador de que la mayoría de los árboles 


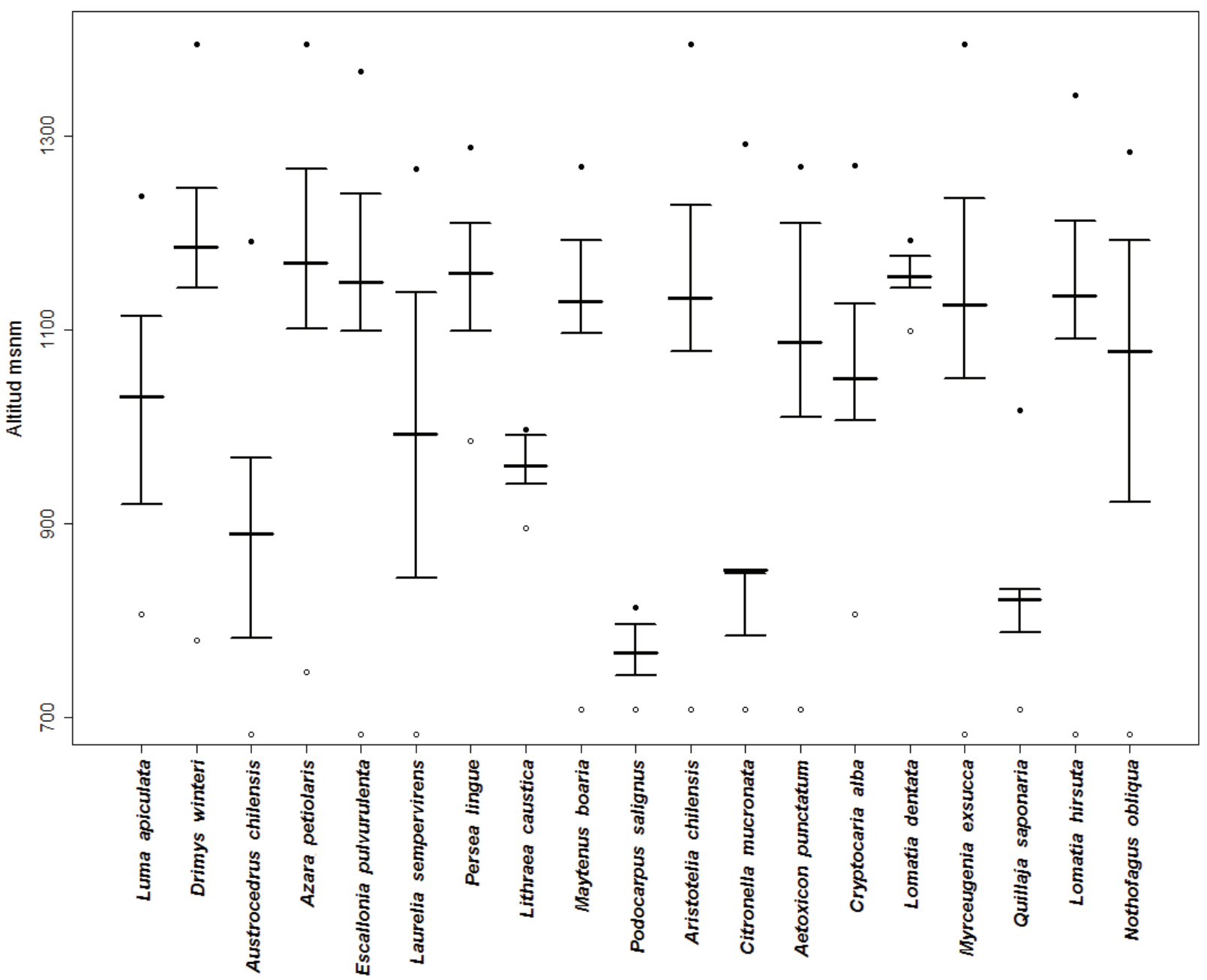

Figura 2. Rango intercuartilíneo de la distribución de altitud (m s.n.m.) en función de las especies. Los puntos negros y blancos representan los valores máximos y mínimos, respectivamente, las barras los rangos intercuartilíneos ( $3^{\text {er }}$ y $1^{\text {er }}$ cuartil) y las líneas horizontales (en negrita) las medias.

Interquartile range of altitudinal distribution ( $\mathrm{m}$ a.s.1.) of the species. The black and white dots depict maximum and minimum values, respectively. Bars depict interquartile range (3rd and 1st quartile) and the central bar depicts medium value.

de C. mucronata estuvieron concentrados y ubicados en zonas que distaron de un comportamiento de valle, presentándose en formaciones de tipo convexas donde las pendientes fueron generalmente homogéneas y muchas veces con magnitudes importantes.

En cuanto a la dispersión de las observaciones del índice de geoforma, respecto a otras especies, C. mucronata tuvo la mayor concentración, donde solo en un 7,1 \% de su amplitud concentró el 50 \% de las observaciones centrales. En el otro extremo se ubicó Drimys winteri con una gran dispersión de las observaciones centrales ocupando un $80 \%$ de su amplitud.

La presencia y abundancia de C. mucronata y su relación con su posición en el paisaje, representada por el índice de geoforma MrVBF mostró un patrón espacial claro (figura 4) destacándose en los sectores altos de la zona de estudio, generalmente, correspondiente a áreas donde no existe formación de valle presentándose además alejada de las principales líneas de drenaje.

Asociación de especies. Citronella mucronata no presentó asociación a la escala de una hectárea con Lomatia hirsuta, Drimys winteri, Myrceugenia exsucca y Escallonia pulverulenta. Considerando la superposición del rango intercuartilíneo de la altitud y el índice de geoforma de las especies, como evidencia para explicar la no coexistencia, se advirtió que $C$. mucronata presentaba una distribución altitudinal superior en su recorrido a Lithraea caustica, Lomatia hirsuta y Escallonia pulverulenta, especies con las cuales no se mezcló (figura 2), y su rango en el índice de geoforma ocupaba terrenos con mucho mayor drenaje (convexas) que Drimys winteri y Myrceugenia exsucca que se posicionaron en terrenos cóncavos (figura 3). En cuanto a las especies con las cuales $C$. mucronata presentó 


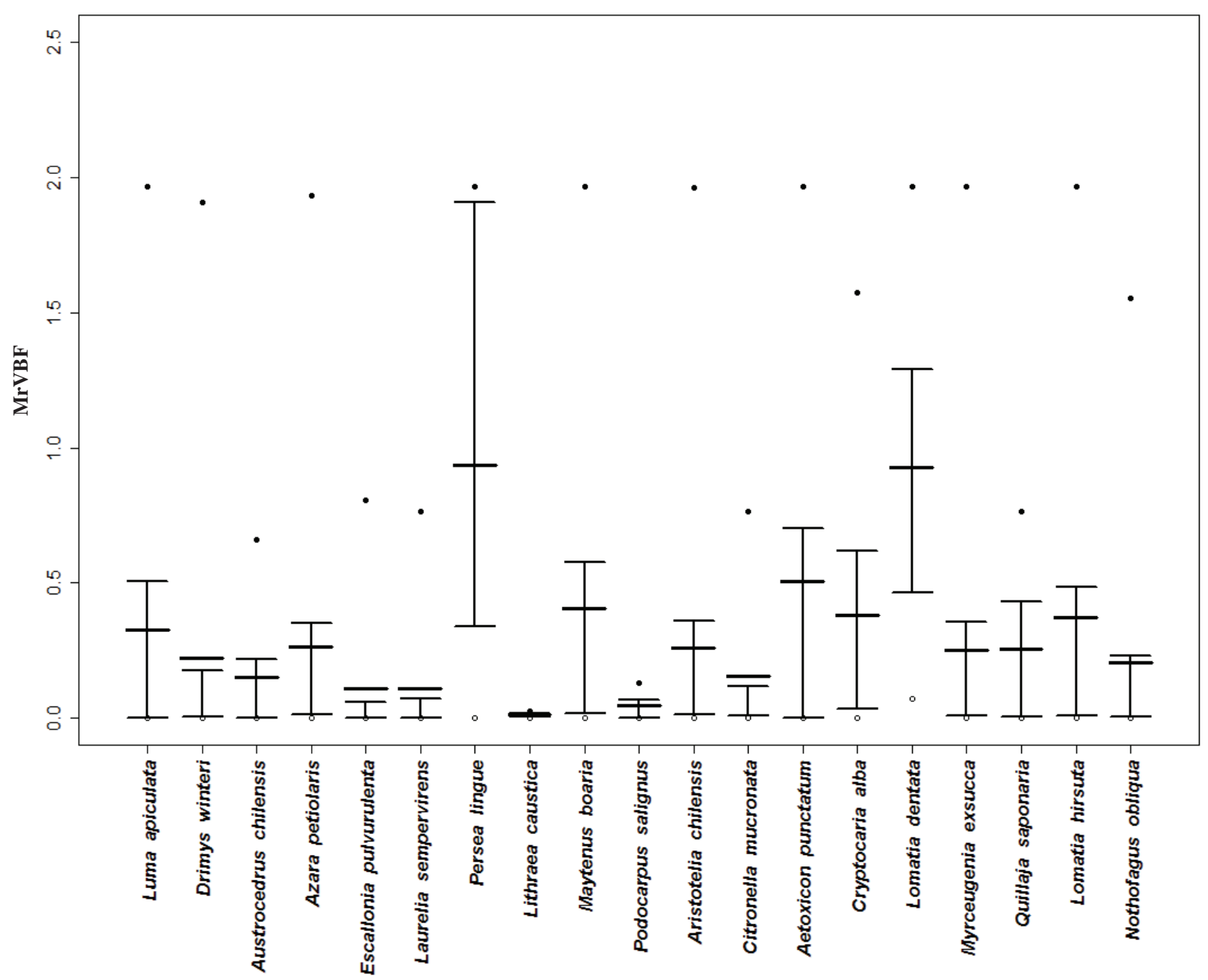

Figura 3. Rango intercuartilíneo del índice de geoforma "Multi-Resolution Valley Bottom Flatness" (MrVBF) de las especies presentes en el área de estudio. Los puntos negros y blancos representan los valores máximos y mínimos, respectivamente, las barras los rangos intercuartilíneos ( $3^{\mathrm{er}}$ y $1^{\text {er }}$ cuartil) y las líneas horizontales (en negrita) las medias.

Interquartile range of the landform index MrVBF ("Multi-Resolution Valley Bottom Flatness") distribution by species. Black and white dots depict maximum and minimum values, respectively. Bars depict the interquartile range (3rd and $1^{\text {st }}$ quartile), and the central bar depicts medium value (in bold).

correlaciones positivas y crecientes en densidad de árboles -medido a través del coeficiente de correlación de Spearman- se relacionó con Persea lingue, Podocarpus salignus, Aextoxicon punctatum, Laurelia sempervirens, Lomatia dentada, Aristotelia chilensis, Luma apiculata, Austrocedrus chilensis y Azara petiolaris y en forma negativa y decreciente con Cryptocarya alba, Maytenus boaria, Quillaja saponaria y Nothofagus obliqua (cuadro 2).

\section{DISCUSIÓN}

En el área de estudio, C. mucronata no puede considerarse escasa o poco frecuente teniendo en cuanta que en la muestra se encontró presente en 31,1 \% sobre una superficie de 1.295 hectáreas superando a 14 de las 17 especies arbóreas registradas. Además C. mucronata ex- hibió una densidad de 117 árboles ha ${ }^{-1}$ (sin considerar la regeneración) que fue superior a 30,8 árboles ha ${ }^{-1}$ (incluida la regeneración) utilizada como referencia por MMA (2012). La presencia de C. mucronata es coincidente con la indicada por el MMA (2012) en cuanto a que se trata de una especie frecuente y con una distribución geográfica relativamente amplia, pero difiere en cuanto a las bajas abundancias poblacionales locales señaladas. Aun cuando los resultados de presencia y abundancia fueron locales, estos provinieron de un área de estudio que contiene una gran variación ambiental (altitud, exposición y drenaje) con especies arbóreas pertenecientes al menos a tres tipos forestales (esclerófilo, roble-hualo y ciprés de la cordillera), representando una gran superficie de interés para el manejo forestal. Desafortunadamente, no existe un parámetro de densidad cuantitativo con el cual contrastarlo. De 


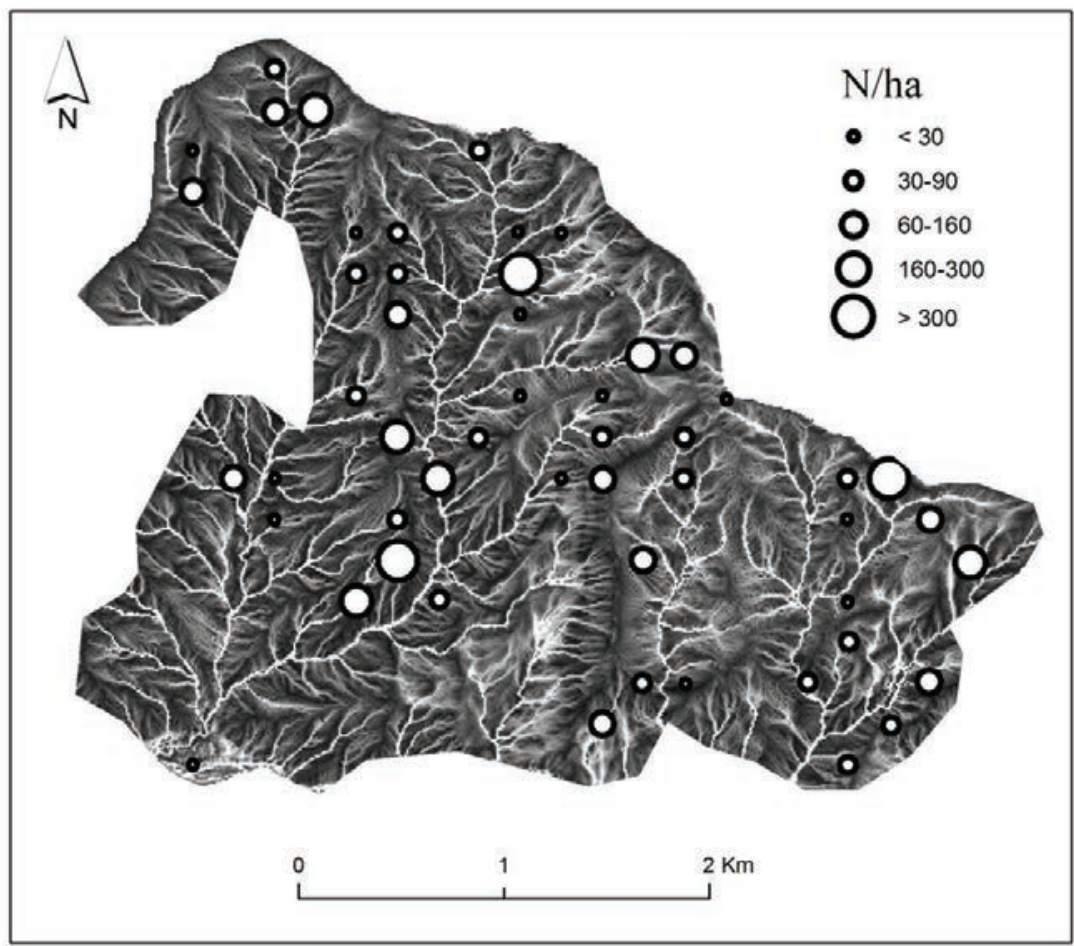

Figura 4. Presencia y abundancia de C. mucronata en la zona de estudio en número de individuos por hectárea $\left(\mathrm{N} \mathrm{ha}^{-1}\right)$ sobre mapa de las líneas de drenaje. drainage lines.

Presence and abundance of Citronella mucronata in the study zone in terms of number of individual per hectare ( $\mathrm{N} \mathrm{ha}^{-1}$ ) over a map of

Cuadro 2. Correlación de Spearman y valor $P$ de la asociación entre Citronella mucronata y otras especies arbóreas a escala de una hectárea.

Spearman correlation among species associated with C. mucronata at the hectare scale.

\begin{tabular}{lcc}
\hline \multicolumn{1}{c}{ Especie } & $\begin{array}{c}\text { Citronella } \\
\text { mucronata }\end{array}$ & Valores $P$ \\
\hline Aextoxicon punctatum & 0,028 & 0,844 \\
Aristotelia chilensis & 0,090 & 0,525 \\
Austrocedrus chilensis & 0,122 & 0,389 \\
Azara petiolaris & 0,140 & 0,321 \\
Cryptocarya alba & $-0,185$ & 0,190 \\
Laurelia sempervirens & 0,045 & 0,751 \\
Lomatia dentata & 0,087 & 0,538 \\
Luma apiculata & 0,118 & 0,404 \\
Maytenus boaria & $-0,133$ & 0,348 \\
Nothofagus obliqua & $-0,002$ & 0,991 \\
Persea lingue & 0,001 & 0,995 \\
Podocarpus salignus & 0,006 & 0,968 \\
Quillaja saponaria & $-0,110$ & 0,435 \\
\hline
\end{tabular}

acuerdo con la última ficha de la especie elaborada por el MMA (2013), este estudio se incluye en el área de distribución de la especie y en el límite oriental de la Cordillera de Los Andes. Un antecedente mencionado en el acta de la sesión ocho del Noveno Proceso de Clasificación de Especies Silvestres da cuenta de la presencia de la especie en forma de rebrotes ocurridos después de talas del bosque nativo original e incluso en plantaciones exóticas. Esta observación es importante y ha sido uno de los argumentos para proponer a la especie en un estado de conservación de Casi Amenazada según la Unión Internacional para la Conservación de la Naturaleza y el Reglamento de Clasificación de Especies (MMA 2012).

La gran mayoría de las especies registradas en el área de estudio presentan una distribución altitudinal coincidentes con la señalada por Donoso et al. (2004) y García y Ormazábal (2008) a excepción de Myrceugenia exsucca y Azara petiolaris que no han sido reportadas. Citronella mucronata no muestra preferencia por las menores altitudes como lo señala MMA (2012); por el contrario, cubre una amplia distribución altitudinal, con preferencia en elevaciones medias y altas, siendo el rango encontrado coincidente con el descrito por Hechenleiter et al. (2005).

El índice de geoforma permite inferir de forma cuantitativa el nivel de concavidad del terreno, siendo un indicador indirecto de la capacidad de retener agua de la su- 
perficie (Taylor et al. 2013). Bajo este supuesto se verifica, en el área de estudio, la preferencia hídrica de las especies hidrófilas por geoformas cóncavas, siendo esta relación coincidente con las descripciones de la literatura (Donoso 1981a, 1993), Caldiz et al. 2004, Donoso et al. 2004, Donoso y Gallo 2004, García et al. 2008). Sin embargo, C. mucronata, tiende a no localizarse en zonas de valle, sino en geoformas de tipo convexas alejadas de las líneas de drenaje y donde las pendientes son generalmente homogéneas y muchas veces con magnitudes importantes. Desde el punto de vista del proceso de formación de suelo, en los lugares donde está presente C. mucronata correspondería a sectores donde la sedimentación se produciría a partir de material localizado en las elevaciones próximas circundantes. Cabe destacar que estos patrones corresponden a sectores altos y bien drenados alejados de quebradas y hábitats húmedos, lo que se aleja de lo señalado por el Ministerio del Medioambiente (MMA 2012). Los resultados del MrVBF de C. mucronata obtenidos de este estudio difieren notablemente de Drimys winteri, Myrceugenia exsucca y Luma apiculata cuyas preferencias hídricas se asocian a terrenos húmedos, propios de terrenos cóncavos y de especies con raíces adaptadas a condiciones de mala aireación por inundación (Donoso 1981a, Caldiz et al. 2004, Donoso et al. 2004). Contraria y aparentemente, C. mucronata tiene preferencias de drenaje similares a las siguientes tres especies esclerófilas: Lithraea caustica cuyo sistema de raíces duales le permite capturar agua a grandes profundidades, lográndose ubicar preferentemente en laderas, a pioneras como Lomatia hirsuta que se ubica en laderas áridas (Donoso 1993), y a Cryptocarya alba, ubicada en condiciones hídricas menos severas que las anteriores logrando además ocupar sitios cóncavos.

En cuanto a la asociatividad, ninguna de las correlaciones resultó significativa, por lo cual se puede indicar que es una especie cuya densidad es relativamente independiente de la densidad con que se presentan las especies con la cuales coexiste.

Donoso (1981b) indica que C. mucronata se ubica en los tipos forestales esclerófilo y roble-hualo, y contrastando sus resultados con este estudio, la especie no se asocia como lo señala con las higrófilas de quebradas del tipo forestal esclerófilo como son Myrceugenia exsucca, Lithraea caustica y Aextoxicon punctatum, y cuando se asocia lo hace negativamente con Cryptocarya alba y Quillaja saponaria. Una excepción la constituye Luma apiculata con la cual se asocia débil y positivamente. En cambio en el tipo forestal roble-hualo, se asocia positivamente con Aristotelia chilensis, Aextoxicon punctatum, Podocarpus salignus y Luma apiculata y negativamente con Nothofagus obliqua, tal como fue señalado por Donoso (1981b).

Si se considera la altitud, el drenaje y la asociatividad con otras especies registradas en el estudio, se advierte que los rodales donde crece $C$. mucronata, se caracterizan por presentar las siguientes situaciones: (1) altitudes cercanas a 1.100 m s.n.m., (2) laderas con alto drenaje y (3) compo- sición de especies asociadas a Persea lingue, Podocarpus salignus, Aextoxicon punctatum, Laurelia sempervirens, Lomatia dentata, Aristotelia chilensis, Luma apiculata, Austrocedrus chilensis, Azara petiolaris, Cryptocarya alba, Maytenus boaria, Quillaja saponaria y Nothofagus obliqua.

En este estudio no se ha considerado la dinámica de la regeneración y crecimiento de las especies ni perturbaciones antrópicas pretéritas como tampoco aspectos de la ecología en cuanto a la competencia, predación o síndrome de dispersión de frutos o semillas los cuales pueden entregar valiosos antecedentes para explicar y entender el patrón de distribución y abundancia en el análisis de C. mucronata.

La anterior clasificación de la IUCN consideró a la C. mucronata como una especie "rara" situación que condicionó de forma importante la intervención de los rodales con su presencia mientras estuvo vigente esa clasificación. Esta situación afectó a los rodales que presentaban las condiciones descritas en las conclusiones del presente trabajo y que de alguna manera deberían ser previstas al momento de cambiar el estatus de conservación.

\section{CONCLUSIONES}

En el área de estudio C. mucronata:

1) Presenta una amplia distribución altitudinal y preferencia de terrenos bien drenados, ubicándose preferentemente en laderas en contraposición a los hábitats húmedos y de baja altitud señalados en la literatura.

2) No se asocia con Lithraea caustica, Lomatia hirsuta, Drimys winteri, Myrceugenia exsucca y Escallonia pulverulenta, cuya distribución altitudinal y posición en el paisaje, según índice de geoforma, difieren respecto a $C$. mucronata.

3) Citronella mucronata no se asocia significativamente con ninguna especie con las cuales coexiste tales como Persea lingue, Podocarpus salignus, Aextoxicon punctatum, Laurelia sempervirens, Lomatia dentada, Aristotelia chilensis, Luma apiculata, Austrocedrus chilensis, Azara petiolaris, Cryptocarya alba, Maytenus boaria, Quillaja saponaria y Nothofagus obliqua.

Los valores de presencia, abundancia, asociatividad y distribución geográfica registrados a micro-escala en la precordillera del Maule, difieren considerablemente de los reportados por el MMA (2012). Se sugiere realizar prospecciones en otras áreas de distribución de la especie con una metodología similar para constatar si solo se trata de un caso particular o simplemente que los argumentos esgrimidos requieren apoyo en una mayor cantidad de información.

\section{REFERENCIAS}

Altamirano A, R Field, L Cayuela, P Aplin, A Lara, JM Rey-Benayas. 2010. Woody species diversity in temperate Andean forests: the need for new conservation strategies. Biological Conservation 143: 2080-2091. 
Altamirano A, P Aplin, A Miranda, L Cayuela, AC Algar, R Field. 2013. High rates of forest loss and turnover obscured by classical landscape measures. Applied Geography 40 (2013) 199-211.

Caldiz MS, AC Prémoli, T Kitzberger. 2004. Variación en Luma apiculata (DC.) Burret. In Donoso C, A Prémoli, L Gallo, R Ipinza eds. Variación intraespecífica en las especies arbóreas de los bosques templados de Chile y Argentina. Santiago, Chile. Editorial Universitaria. p. 381-389.

CIREN (Centro de Información de Recursos Naturales, CL). 1996. Estudio Agrológico VI Región. Descripciones de suelos, materiales y símbolos. Publicación nº114. Santiago, Chile. 479 p.

CONAF (Corporación Nacional Forestal, CL). 1989. Libro Rojo de la Flora Terrestre de Chile. República de Chile Ministerio de Agricultura. Santiago, Chile. Corporación Nacional Forestal. 157 p.

CONAMA (Comisión Nacional del Medio Ambiente, CL). 1994. Ley 119.300: Bases generales del medio ambiente. Consultada 31 oct. 2012 Disponible en http://www.sinia.cl/1292/ articles-26087_ley_bases.pdf

Donoso C. 1981a. Ecología forestal. El bosque y su medioambiente. Santiago, Chile. Editorial Universitaria. 369 p.

Donoso C. 1981b. Tipos forestales de los bosques nativos de Chile. Documento de Trabajo No 38. Investigación y desarrollo forestal (CONAF, PNUD, FAO) (Publicación FAO Chile). $70 \mathrm{p}$.

Donoso C. 1993. Bosques templados de Chile y Argentina. Variación, estructura y dinámica. Santiago, Chile. Editorial Universitaria. $484 \mathrm{p}$.

Donoso C, C Navarro, M Hernández. 2004. Variación en Drimys winteri J.R. et G. Forster. In Donoso C, A Prémoli, L Gallo, R Ipinza eds. Variación intraespecífica en las especies arbóreas de los bosques templados de Chile y Argentina. Santiago, Chile. Editorial Universitaria. p. 303-320.

Donoso C, L Gallo. 2004. Aspectos conceptuales y metodológicos. In Donoso C, A Prémoli, L Gallo, R Ipinza eds. Variación intraespecífica en las especies arbóreas de los bosques templados de Chile y Argentina. Santiago, Chile. Editorial Universitaria. p. 23-37.

D’Ottone H. 1971. Estadística elemental. Santiago, Chile. Cooperativa de Cultura y Publicaciones, Ltda. 407 p.

Gajardo R. 1994. La vegetación natural de Chile. Clasificación y distribución geográfica. Santiago, Chile. Editorial Universitaria. 165 p.

Gallant JC, TI Dowling. 2003. A multiresolution index of valley bottom flatness for mapping depositional areas. Water Resources Research 39 (12): 1347-1359.
García N, C Ormazábal. 2008. Árboles nativos de Chile. Santiago, Chile. Enersis S.A. 196 p.

Hechenleitner P, M Gardner, P Thomas, C Echeverría, B Escobar, P Brownless, C Martínez. 2005. Plantas amenazadas del centro-sur de Chile. Distribución, conservación y propagación. Primera Edición. Valdivia, Chile. Universidad Austral de Chile y Real Jardín Botánico de Edimburgo. 188 p.

Hoersch B, G Braun, U Schmid. 2002. Relation between landform and vegetation in alpine regions of Wallis, Switzerland. A multiscale remote sensing and GIS approach. Computers, Environment and Urban Systems 26(2-3):113-139.

Luebert F, P Pliscoff. 2006. Sinopsis bioclimática y vegetacional de Chile. Santiago, Chile. Editorial Universitaria. 296 p.

MMA (Ministerio del Medio Ambiente, CL). 2012. Lista de especies nativas según estado de conservación. Consultado 31 oct. 2012. Disponible en http://www.mma.gob.cl/clasificacionespecies

MMA (Ministerio del Medio Ambiente, CL). 2013. Acta Sesión No 08 Noveno Proceso Clasificación. Consultado 31 Mayo 2013. Disponible en http://www.mma.gob.cl/clasificacionespecies/Anexo_noveno_proceso/Acta_RCE_8_NOVENO_Proc_27_diciembre_2012.pdf

Newton AC, C Echeverría, E Cantarello, G Bolados. 2011. Projecting impacts of human disturbances to inform conservation planning and management in a dryland forest landscape. Biological Conservation 144 (2011) 1949-1960.

Santibáñez F, JM Uribe.1993. Atlas agroclimático de Chile: Regiones Sexta, Séptima y Octava. CORFO, Fondo de Investigación Agropecuaria. Ministerio de Agricultura. 99 p.

Taylor J, F Jacob, M Galleguillos, L Prévot, N Guix, P Lagacherie. 2013. The utility of remotely-sensed vegetative and terrain covariates at different spatial resolutions in modelling soil and watertable depth (for digital soil mapping). Geoderma 193-194: 83-93.

Teng L, J Vaze, NK Tuteja, JC Gallant. 2005. CLASS Spatial Analyst: A GIS based tool for distributed hydrological modelling. In Zerger A, RM Argent eds. International Congress on Modelling and Simulation. Modelling and Simulation Society of Australia and New Zealand. p. 1485-1491.

Teng L, J Vaze, NK Tuteja, JC Gallant. 2008. A GIS-based tool for spatial and distributed hydrological modelling: CLASS Spatial Analyst. Transaction in GIS 12(2): 209-225.

Weinacker H, B Koch, U Heyder, R Weinacker. 2004. TREESVIS-A software system for simultaneous 3D-real-time visualisation of DTM, DSM, laser raw data, multispectral data, simple tree and building models. International Archives of Photogrammetry, Remote Sensing and Spatial Information Sciences 36: 90-95. 